\title{
The Principle of Competitive Exclusion about a Stochastic Lotka-Volterra Model with Two Predators Competing for One Prey
}

\author{
Zhongwei Cao $\mathbb{D}^{1},{ }^{1}$ Qiumei Zhang ${ }^{\mathbb{D}}{ }^{2}$, and Yanan Zhao ${ }^{2}$ \\ ${ }^{1}$ Department of Applied Mathematics, Jilin University of Finance and Economics, Changchun 130117, China \\ ${ }^{2}$ School of Science, Changchun University, Changchun 130022, China \\ Correspondence should be addressed to Qiumei Zhang; zhangqm1110@163.com
}

Received 12 March 2018; Revised 29 June 2018; Accepted 9 July 2018; Published 17 July 2018

Academic Editor: Rodica Luca

Copyright (C) 2018 Zhongwei Cao et al. This is an open access article distributed under the Creative Commons Attribution License, which permits unrestricted use, distribution, and reproduction in any medium, provided the original work is properly cited.

This paper concerns a stochastic Lotka-Volterra model with two predators competing for one prey. The sufficient conditions which guarantee the principle of competitive exclusion for this perturbed model are given by using Lyapunov analysis methods. Numerical simulations for a set of parameter values are presented to illustrate the analytical findings.

\section{Introduction}

Volterra has argued that the coexistence of two or more predators competing for fewer prey resources is impossible, which was later known as the principle of competitive exclusion. The principle of competitive exclusion was reexamined by Koch [1] in 1974 who found via numerical simulation that the coexistence of two predators competing exploitatively for a single prey species in a constant and uniform environment was in fact possible when the predator functional response to the prey density was assumed according to nonlinear function, and such coexistence occurred along what appeared to be a periodic orbit in the positive octant of $R^{3}$ rather than an equilibrium. The similar themes were discussed in [2-8]. The authors in [6] studied the global dynamics of 3-dimensional Lotka-Volterra models with two predators competing for a single prey species in a constant and uniform environment. They obtained sufficient and necessary conditions for the principle of competitive exclusion to hold and gave the global dynamical behavior of the three species. They assumed that the two predator species compete purely exploitatively with no interference between rivals, the growth rate of the prey species is logistic or linear in the absence of predation respectively, and the predator's functional response is linear.
Based on the above assumptions the model can be written as follows:

$$
\begin{aligned}
& \frac{d S(t)}{d t}=S(t)\left(b_{1}-a_{11} S(t)-a_{12} X(t)-a_{13} Y(t)\right), \\
& \frac{d X(t)}{d t}=X(t)\left(-b_{2}+b_{21} S(t)\right), \\
& \frac{d Y(t)}{d t}=Y(t)\left(-b_{3}+b_{31} S(t)\right),
\end{aligned}
$$

where $S(t), X(t), Y(t)$ are the densities of the prey and the $i$ th predator $(\mathrm{i}=1,2)$ population, respectively. $b_{1}$ is the intrinsic rate of growth of the prey, and $1 / a_{11}$ is the carrying capacity of the prey, which describes the richness of resources for prey. $a_{12}, a_{13}$ are the effects of the $i$ th predation on the prey, $b_{2}, b_{3}$ are the natural death rates of the $i$ th predator in the absence of prey, and $b_{21}, b_{31}$ are the efficiency and propagation rates of the $i$ th predator in the presence of prey.

The above discussion rests on the assumption that the environmental parameters involved with the model system are all constants irrespective to time and environmental fluctuations. We consider the effect of environmental fluctuation on the model system. There are two ways to develop the stochastic model corresponding to an existing deterministic 
one. Firstly, one can replace the environmental parameters involved with the deterministic model system by some random parameters; see $[9,10]$. Secondly, one can add the randomly fluctuating driving force directly to the deterministic growth equations of prey and predator populations without altering any particular parameter; see [11-14]. In the present study we follow the second method. To incorporate the effect of randomly fluctuating environment, we introduce stochastic perturbation terms in the growth equations of both prey and predator population:

$$
\begin{aligned}
d S(t)= & S(t)\left(b_{1}-a_{11} S(t)-a_{12} X(t)-a_{13} Y(t)\right) d t \\
& +\sigma_{1} S(t) d B_{1}(t), \\
d X(t)= & X(t)\left(-b_{2}+b_{21} S(t)\right) d t+\sigma_{2} X(t) d B_{2}(t), \\
d Y(t)= & Y(t)\left(-b_{3}+b_{31} S(t)\right) d t+\sigma_{3} Y(t) d B_{3}(t),
\end{aligned}
$$

where $B_{1}(t), B_{2}(t), B_{3}(t)$ are independent Brownian motions defined on a complete probability space $\left(\Omega, \mathscr{F},\left\{\mathscr{F}_{t}\right\}_{t \geq 0}, \mathbb{P}\right)$ with a filtration $\left\{\mathscr{F}_{t}\right\}_{t \geq 0}$ satisfying the usual conditions, and $\sigma_{1}^{2}, \sigma_{2}^{2}, \sigma_{3}^{2}$ are the intensities of environmental white noise.

The aim is to study the dynamics of 3-dimensional LotkaVolterra models with two predators competing for a single prey species by stochastic perturbation. Zhang and Jiang [15] give the sufficient conditions which guarantee that the principle of coexistence holds for this perturbed model via Markov semigroup theory. Furthermore, they prove that the densities of the solution can converge in $L^{1}$ to an invariant density under appropriate conditions. In this paper, we study the principle of competitive exclusion associated with system (2). The paper is organized as follows. In Section 2, we give the sufficient conditions to guarantee that the principle of competitive exclusion holds for system (2). We make simulations to illustrate our analytical results in Section 3.

Remark 1. In [15] (Theorem 2.1), Zhang and Jiang show that, for any initial value $(S(0), X(0), Y(0)) \in R_{+}^{3}$, system (2) has a unique positive solution $(S(t), X(t), Y(t))$ a.s.

\section{The Principle of Competitive Exclusion for System (2)}

In this section, we show that system (2) allows the competitive exclusion of two competing predators for some values of parameters, which implies that the competitive exclusion of two predators competing for a single prey species is possible when the predator functional response to the prey density is linear.

For simplicity, define

$$
\langle x(t)\rangle=\frac{1}{t} \int_{0}^{t} x(r) d r .
$$

Lemma 2. Assume $\left(b_{2} \wedge b_{3}\right)>(1 / 2)\left(\sigma_{2}^{2} \vee \sigma_{3}^{2}\right)$. Then for any initial value $(S(0), X(0), Y(0)) \in R_{+}^{3}$, the solution of system (2) has the property

$$
\begin{aligned}
& \limsup _{t \rightarrow \infty} \frac{\log \left[S(t)+\left(a_{12} / b_{21}\right) X(t)+\left(a_{13} / b_{31}\right) Y(t)\right]}{\log t} \\
& \quad \leq \frac{1}{\theta} \quad \text { a.s. }
\end{aligned}
$$

where $\theta$ is a positive constant that satisfies $2<\theta<1+2\left(\left(b_{2} \wedge\right.\right.$ $\left.\left.b_{3}\right) /\left(\sigma_{2}^{2} \vee \sigma_{3}^{2}\right)\right)$.

Proof. Let

$$
\begin{aligned}
U(t) & =S(t)+\frac{a_{12}}{b_{21}} X(t)+\frac{a_{13}}{b_{31}} Y(t) \\
& :=S(t)+X_{1}(t)+Y_{1}(t) .
\end{aligned}
$$

By Itô's formula and (2), we have

$$
\begin{aligned}
d U(t)= & {\left[S(t)\left(b_{1}-a_{11} S(t)\right)-b_{2} X_{1}(t)-b_{3} Y_{1}(t)\right] d t } \\
& +\sigma_{1} S(t) d B_{1}(t)+\sigma_{2} X_{1}(t) d B_{2}(t) \\
& +\sigma_{3} Y_{1}(t) d B_{3}(t) .
\end{aligned}
$$

Define the function $W$ by $W(U)=(1+U)^{\theta}$. Then

$$
\begin{aligned}
& d W(U)=L W(U) d t+\theta(1+U(t))^{\theta-1}\left[\sigma_{1} S(t) d B_{1}(t)\right. \\
& \left.+\sigma_{2} X_{1}(t) d B_{2}(t)+\sigma_{3} Y_{1}(t) d B_{3}(t)\right]
\end{aligned}
$$

where

$$
\begin{aligned}
& L W(U)=\theta(1+U)^{\theta-1}\left[S\left(b_{1}-a_{11} S\right)-b_{2} X_{1}-b_{3} Y_{1}\right] \\
& +\frac{\theta(\theta-1)}{2}(1+U)^{\theta-2}\left(\sigma_{1}^{2} S^{2}+\sigma_{2}^{2} X_{1}^{2}+\sigma_{3}^{2} Y_{1}^{2}\right)=\theta(1 \\
& +U)^{\theta-2}\left\{(1+U)\left[S\left(b_{1}-a_{11} S\right)-b_{2} X_{1}-b_{3} Y_{1}\right]\right. \\
& \left.+\frac{\theta-1}{2}\left(\sigma_{1}^{2} S^{2}+\sigma_{2}^{2} X_{1}^{2}+\sigma_{3}^{2} Y_{1}^{2}\right)\right\} \leq \theta(1+U)^{\theta-2} \\
& +\left\{(1+S+Z)\left[b_{1} S-a_{11} S^{2}-\left(b_{2} \wedge b_{3}\right) Z\right]\right. \\
& \left.\left.+\frac{\theta-1}{2}\left[\sigma_{1}^{2} S^{2}+\left(\sigma_{2}^{2} \vee \sigma_{3}^{2}\right) Z^{2}\right)\right]\right\} \leq \theta(1+U)^{\theta-2} \\
& +\left\{-a_{11} S^{3}+\left(b_{1}+\frac{\theta-1}{2} \sigma_{1}^{2}\right) S^{2}+b_{1} S Z+b_{1} S\right. \\
& \left.-\left[\left(b_{2} \wedge b_{3}\right)-\frac{\theta-1}{2}\left(\sigma_{2}^{2} \vee \sigma_{3}^{2}\right)\right] Z^{2}\right\} ;
\end{aligned}
$$

here $Z=X_{1}+Y_{1}$.

Now, choose a constant $\lambda>0$ sufficiently small such that it satisfies

$$
\left(b_{2} \wedge b_{3}\right)-\frac{\theta-1}{2}\left(\sigma_{2}^{2} \vee \sigma_{3}^{2}\right)-\frac{3 \lambda}{\theta} \gg 0 .
$$

Applying Itô's formula, we have

$$
\begin{aligned}
E\left[e^{\lambda t} W(U(t))\right]= & W(U(0)) \\
& +E \int_{0}^{t} L\left[e^{\lambda s} W(U(s))\right] d s,
\end{aligned}
$$


where

$$
\begin{aligned}
L & {\left[e^{\lambda t} W(U)\right]=\lambda e^{\lambda t} W(U(t))+e^{\lambda t} L W(U) \leq \theta e^{\lambda t}(1} \\
& +U)^{\theta-2}\left\{-a_{11} S^{3}+\left(b_{1}+\frac{\theta-1}{2} \sigma_{1}^{2}\right) S^{2}+b_{1} S Z+b_{1} S\right. \\
& -\left[\left(b_{2} \wedge b_{3}\right)-\frac{\theta-1}{2}\left(\sigma_{2}^{2} \vee \sigma_{3}^{2}\right)\right] Z^{2} \\
& \left.+\frac{\lambda}{\theta}(1+S+Z)^{2}\right\} \leq \theta e^{\lambda t}(1+U)^{\theta-2}\left\{-a_{11} S^{3}\right. \\
& +\left(b_{1}+\frac{\theta-1}{2} \sigma_{1}^{2}+\frac{3 \lambda}{\theta}\right) S^{2}+b_{1} S Z+b_{1} S+\frac{3 \lambda}{\theta} \\
& \left.-\left[\left(b_{2} \wedge b_{3}\right)-\frac{\theta-1}{2}\left(\sigma_{2}^{2} \vee \sigma_{3}^{2}\right)-\frac{3 \lambda}{\theta}\right] Z^{2}\right\} \\
& \leq \theta e^{\lambda t}(1+U)^{\theta-2}\left\{-a_{11} S^{3}+c S^{2}+b_{1} S+\frac{3 \lambda}{\theta}\right. \\
& \left.-\frac{1}{2}\left[\left(b_{2} \wedge b_{3}\right)-\frac{\theta-1}{2}\left(\sigma_{2}^{2} \vee \sigma_{3}^{2}\right)-\frac{3 \lambda}{\theta}\right] Z^{2}\right\} \\
& \leq \theta e^{\lambda t} H ;
\end{aligned}
$$

here $c:=b_{1}+((\theta-1) / 2) \sigma_{1}^{2}+3 \lambda / \theta+(1 / 2) b_{1}^{2}\left[\left(b_{2} \wedge b_{3}\right)-\right.$ $\left.((\theta-1) / 2)\left(\sigma_{2}^{2} \vee \sigma_{3}^{2}\right)-3 \lambda / \theta\right]^{-1}$ and $H:=\sup _{(S, Z) \in R_{+}^{2}}(1+S+$
$Z)^{\theta-2}\left\{-a_{11} S^{3}+c S^{2}+b_{1} S+3 \lambda / \theta-(1 / 2)\left[\left(b_{2} \wedge b_{3}\right)-((\theta-1) / 2)\left(\sigma_{2}^{2} \vee\right.\right.\right.$ $\left.\left.\left.\sigma_{3}^{2}\right)-3 \lambda / \theta\right] Z^{2}\right\}$. This implies

$$
E\left[e^{\lambda t}(1+U)^{\theta}\right] \leq(1+U(0))^{\theta}+\frac{\theta e^{\lambda t} H}{\lambda} .
$$

Then

$$
\limsup _{t \rightarrow \infty} E\left[(1+U)^{\theta}\right] \leq \frac{\theta H}{\lambda} .
$$

There exists a positive constant $M$ such that

$$
E\left[(1+U)^{\theta}\right] \leq M, \quad t \geq 0 .
$$

For sufficiently small $\delta>0, k=1,2, \ldots,(7)$ implies that

$$
\begin{aligned}
& E\left[\sup _{(k-1) \delta \leq t \leq k \delta}(1+U(t))^{\theta}\right] \\
& \quad \leq E\left[(1+U((k-1) \delta))^{\theta}\right]+I_{1}+I_{2} \leq M+I_{1}+I_{2},
\end{aligned}
$$

where

$$
\begin{aligned}
I_{1} & =E\left[\sup _{(k-1) \delta \leq t \leq k \delta} \int_{(k-1) \delta}^{t} \theta(1+U(s))^{\theta-2}\right. \\
& \left.\cdot\left\{-a_{11} S^{3}(s)+\left(b_{1}+\frac{\theta-1}{2} \sigma_{1}^{2}\right) S^{2}(s)+b_{1} S(s) Z(s)+b_{1} S(s)-\left[\left(b_{2} \wedge b_{3}\right)-\frac{\theta-1}{2}\left(\sigma_{2}^{2} \vee \sigma_{3}^{2}\right)\right] Z^{2}(s)\right\} d s\right] \\
& \leq E\left[\sup _{(k-1) \delta \leq t \leq k \delta} \int_{(k-1) \delta}^{t} \theta(1+U(s))^{\theta-2}\right. \\
& \left.\cdot\left\{-a_{11} S^{3}(s)+\left(b_{1}+\frac{\theta-1}{2} \sigma_{1}^{2}+\frac{1}{2} b_{1}^{2}\left[\left(b_{2} \wedge b_{3}\right)-\frac{\theta-1}{2}\left(\sigma_{2}^{2} \vee \sigma_{3}^{2}\right)\right]^{-1}\right) S^{2}(s)+b_{1} S(s)-\frac{1}{2}\left[\left(b_{2} \wedge b_{3}\right)-\frac{\theta-1}{2}\left(\sigma_{2}^{2} \vee \sigma_{3}^{2}\right)\right] Z^{2}(s)\right\} d s\right] \\
& \leq \theta H_{0} E\left[\sup _{(k-1) \delta \leq t \leq k \delta} \int_{(k-1) \delta}^{t}(1+U(s))^{\theta} d s\right] \leq \theta H_{0} E\left[\int_{(k-1) \delta}^{k \delta}(1+U(s))^{\theta} d s\right] \leq \theta H_{0} \delta E\left[\sup _{(k-1) \delta \leq t \leq k \delta}(1+U(t))^{\theta}\right] ;
\end{aligned}
$$

here $H_{0}:=\sup _{(S, Z) \in R_{+}^{2}}\left\{-a_{11} S^{3}(s)+\left(b_{1}+((\theta-1) / 2) \sigma_{1}^{2}+\right.\right.$ $\left.(1 / 2) b_{1}^{2}\left[\left(b_{2} \wedge b_{3}\right)-((\theta-1) / 2)\left(\sigma_{2}^{2} \vee \sigma_{3}^{2}\right)\right]^{-1}\right) S^{2}(s)+b_{1} S(s)-$ $\left.(1 / 2)\left[\left(b_{2} \wedge b_{3}\right)-((\theta-1) / 2)\left(\sigma_{2}^{2} \vee \sigma_{3}^{2}\right)\right] Z^{2}(s)\right\}$, and

$$
\begin{aligned}
I_{2} & =E\left[\sup _{(k-1) \delta \leq t \leq k \delta}\left|\int_{(k-1) \delta}^{t} \theta(1+U(t))^{\theta-1}\left(\sigma_{1} S(s) d B_{1}(s)+\sigma_{2} X_{1}(s) d B_{2}(s)+\sigma_{3} Y_{1}(s) d B_{3}(s)\right)\right|\right] \\
& \leq \sqrt{32} E\left[\int_{(k-1) \delta}^{k \delta} \theta^{2}(1+U(s))^{2(\theta-1)}\left(\sigma_{1}^{2} S^{2}(s)+\sigma_{2}^{2} X_{1}^{2}(s)+\sigma_{3}^{2} Y_{1}^{2}(s)\right) d s\right]^{1 / 2}
\end{aligned}
$$




$$
\begin{aligned}
& \leq \sqrt{32} \theta\left(\sigma_{1}^{2} \vee \sigma_{2}^{2} \vee \sigma_{3}^{2}\right)^{1 / 2} \delta^{1 / 2} E\left[\sup _{(k-1) \delta \leq t \leq k \delta}(1+U(t))^{2 \theta}\right]^{1 / 2} \\
& \leq \sqrt{32} \theta\left(\sigma_{1}^{2} \vee \sigma_{2}^{2} \vee \sigma_{3}^{2}\right)^{1 / 2} \delta^{1 / 2} E\left[\sup _{(k-1) \delta \leq t \leq k \delta}(1+U(t))^{\theta}\right]
\end{aligned}
$$

according to the Burkholder-Davis-Gundy inequality. Therefore,

$$
\begin{aligned}
& E\left[\sup _{(k-1) \delta \leq t \leq k \delta}(1+U(t))^{\theta}\right] \leq E\left[(1+U((k-1) \delta))^{\theta}\right] \\
& +\left[\theta H_{0} \delta+\sqrt{32} \theta\left(\sigma_{1}^{2} \vee \sigma_{2}^{2} \vee \sigma_{3}^{2}\right)^{1 / 2} \delta^{1 / 2}\right] \\
& \cdot E\left[\sup _{(k-1) \delta \leq t \leq k \delta}(1+U(t))^{\theta}\right] .
\end{aligned}
$$

In particular, choose $\delta>0$ such that $\theta H_{0} \delta+\sqrt{32} \theta\left(\sigma_{1}^{2} \vee \sigma_{2}^{2} \vee\right.$ $\left.\sigma_{3}^{2}\right)^{1 / 2} \delta^{1 / 2} \leq 1 / 2$, and so

$$
\begin{aligned}
& E\left[\sup _{(k-1) \delta \leq t \leq k \delta}(1+U(t))^{\theta}\right] \\
& \quad \leq 2 E\left[(1+U((k-1) \delta))^{\theta}\right] \leq 2 M .
\end{aligned}
$$

Let $\varepsilon>0$ be arbitrary. Then, by the Chebyshev's inequality, we have

$$
\begin{array}{r}
P\left\{\omega: \sup _{(k-1) \delta \leq t \leq k \delta}(1+U(t))^{\theta}>(k \delta)^{1+\varepsilon}\right\} \leq \\
\quad \frac{2 M}{(k \delta)^{1+\varepsilon}}, \\
k=1,2, \ldots
\end{array}
$$

In view of the Borel-Cantelli lemma, we see that, for almost all $\omega \in \Omega$,

$$
\sup _{(k-1) \delta \leq t \leq k \delta}(1+U(t))^{\theta} \leq(k \delta)^{1+\varepsilon}
$$

holds for all but finitely many $k$. Hence there exists an integer $k_{0}(\omega)>1 / \delta+2$, for almost all $\omega \in \Omega$, for which (21) holds whenever $k \geq k_{0}$. Consequently, for almost all $\omega \in \Omega$, if $k \geq$ $k_{0}$ and $(k-1) \delta \leq t \leq k \delta$,

$$
\frac{\log (1+U(t))^{\theta}}{\log t} \leq \frac{(1+\varepsilon) \log (k \delta)}{\log ((k-1) \delta)} .
$$

Therefore,

$$
\limsup _{t \rightarrow \infty} \frac{\log (1+U(t))^{\theta}}{\log t} \leq 1+\varepsilon \quad \text { a.s. }
$$

Letting $\varepsilon \longrightarrow 0$, we obtain the desired assertion

$$
\limsup _{t \rightarrow \infty} \frac{\log (1+U(t))^{\theta}}{\log t} \leq 1 \quad \text { a.s. }
$$

and

$$
\limsup _{t \rightarrow \infty} \frac{\log U(t)}{\log t} \leq \limsup _{t \longrightarrow \infty} \frac{\log (1+U(t))}{\log t} \leq \frac{1}{\theta} \quad \text { a.s. }
$$

Hence the proof of this lemma is complete.

Theorem 3. Let $(S(t), X(t), Y(t))$ be the solution of system (2) with initial value $(S(0), X(0), Y(0)) \in R_{+}^{3}$. Assume $\left(b_{2} \wedge b_{3}\right)>$ $(1 / 2)\left(\sigma_{2}^{2} \vee \sigma_{3}^{2}\right)$. The principle of competitive exclusion holds for system (2) if one of the following conditions holds.

(i) If $b_{1}>(1 / 2) \sigma_{1}^{2}, b_{3} / b_{31} \geq b_{2} / b_{21},\left(b_{3}+(1 / 2) \sigma_{3}^{2}\right) / b_{31}>$ $b_{2} / b_{21}+\left(K(\sigma) / a_{11}\right)^{1 / 2}$ and $\left(b_{1}-(1 / 2) \sigma_{1}^{2}\right) / a_{11}>\left(b_{2}+\right.$ $\left.(1 / 2) \sigma_{2}^{2}\right) / b_{21}$, then

$$
\begin{aligned}
& \liminf _{t \rightarrow \infty}\langle X(t)\rangle \geq \frac{1}{a_{12}}\left[b_{1}-\frac{1}{2} \sigma_{1}^{2}-a_{11} \frac{b_{2}+(1 / 2) \sigma_{2}^{2}}{b_{21}}\right] \\
& >0 \quad \text { a.s. } \\
& \underset{t \rightarrow \infty}{\lim \sup _{t \rightarrow \infty}} \frac{\log Y(t)}{t} \\
& \quad \leq-b_{3}-\frac{1}{2} \sigma_{3}^{2}+\frac{b_{31} b_{2}}{b_{21}}+b_{31}\left(\frac{K(\sigma)}{a_{11}}\right)^{1 / 2}<0 \text { a.s. }
\end{aligned}
$$

where $K(\sigma)=\bar{S} \sigma_{1}^{2} / 2+a_{12} \bar{X} \sigma_{2}^{2} / 2 b_{21}$ and $(\bar{S}, \bar{X}, 0)$ is the boundary equilibrium of system (1).

(ii) If $b_{1}>(1 / 2) \sigma_{1}^{2}, b_{2} / b_{21} \geq b_{3} / b_{31},\left(b_{2}+(1 / 2) \sigma_{2}^{2}\right) / b_{21}>$ $b_{3} / b_{31}+\left(K_{0}(\sigma) / a_{11}\right)^{1 / 2}$ and $\left(b_{1}-(1 / 2) \sigma_{1}^{2}\right) / a_{11}>\left(b_{3}+\right.$ $\left.(1 / 2) \sigma_{3}^{2}\right) / b_{31}$, then

$$
\begin{aligned}
& \underset{t \rightarrow \infty}{\lim \sup } \frac{\log X(t)}{t} \\
& \quad \leq-b_{2}-\frac{1}{2} \sigma_{2}^{2}+\frac{b_{21} b_{3}}{b_{31}}+b_{21}\left(\frac{K_{0}(\sigma)}{a_{11}}\right)^{1 / 2}<0 \quad \text { a.s. }
\end{aligned}
$$




$$
\begin{aligned}
& \liminf _{t \rightarrow \infty}\langle Y(t)\rangle \geq \frac{1}{a_{13}}\left[b_{1}-\frac{1}{2} \sigma_{1}^{2}-a_{11} \frac{b_{3}+(1 / 2) \sigma_{3}^{2}}{b_{31}}\right] \\
& >0 \quad \text { a.s. }
\end{aligned}
$$

where $K_{0}(\sigma)=\widetilde{S} \sigma_{1}^{2} / 2+a_{13} \tilde{Y} \sigma_{3}^{2} / 2 b_{31}$ and $(\widetilde{S}, 0, \tilde{Y})$ is the boundary equilibrium of system (1).

Proof. (i) If $\left(b_{1}-(1 / 2) \sigma_{1}^{2}\right) / a_{11}>\left(b_{2}+(1 / 2) \sigma_{2}^{2}\right) / b_{21}$, system (1) has the boundary equilibrium $(\bar{S}, \bar{X}, 0)$. Then

$$
\begin{aligned}
& \bar{S}=\frac{b_{2}}{b_{21}}, \\
& \bar{X}=\frac{b_{1} b_{21}-a_{11} b_{2}}{a_{12} b_{21}} .
\end{aligned}
$$

Define

$$
\begin{aligned}
V(S, X, Y)= & S-\bar{S}-\bar{S} \log \frac{S}{\bar{S}} \\
& +\frac{a_{12}}{b_{21}}\left(X-\bar{X}-\bar{X} \log \frac{X}{\bar{X}}\right)+\frac{a_{13}}{b_{31}} Y .
\end{aligned}
$$

By Itồs formula and (2), we have

$$
\begin{aligned}
d V & =\left\{-a_{11}(S-\bar{S})^{2}-a_{12}(S-\bar{S})(X-\bar{X})\right. \\
& \left.-a_{13}(S-\bar{S}) Y+\frac{\bar{S} \sigma_{1}^{2}}{2}\right\} d t+\sigma_{1}(S-\bar{S}) d B_{1}(t) \\
& +\frac{a_{12}}{b_{21}}\left\{\left[b_{21}(S-\bar{S})(X-\bar{X})+\frac{\bar{X} \sigma_{2}^{2}}{2}\right] d t\right. \\
& \left.+\sigma_{2}(X-\bar{X}) d B_{2}(t)\right\} \\
& +\frac{a_{13}}{b_{31}}\left\{\left[\left(-b_{3}+b_{31} \bar{S}\right) Y+b_{31}(S-\bar{S}) Y\right] d t\right. \\
& \left.+\sigma_{3} Y d B_{3}(t)\right\}:=L V d t+\sigma_{1}(S-\bar{S}) d B_{1}(t)+\frac{a_{12}}{b_{21}} \\
& \cdot \sigma_{2}(X-\bar{X}) d B_{2}(t)+\frac{a_{13}}{b_{31}} \sigma_{3} Y d B_{3}(t),
\end{aligned}
$$

where

$$
\begin{aligned}
L V= & -a_{11}(S-\bar{S})^{2}-a_{12}(S-\bar{S})(X-\bar{X}) \\
& -a_{13}(S-\bar{S}) Y+\frac{\bar{S} \sigma_{1}^{2}}{2} \\
& +\frac{a_{12}}{b_{21}}\left[b_{21}(S-\bar{S})(X-\bar{X})+\frac{\bar{X} \sigma_{2}^{2}}{2}\right] \\
& +\frac{a_{13}}{b_{31}}\left[\left(-b_{3}+b_{31} \bar{S}\right) Y+b_{31}(S-\bar{S}) Y\right] \\
= & -a_{11}(S-\bar{S})^{2}+\frac{\bar{S} \sigma_{1}^{2}}{2}+\frac{a_{12} \bar{X} \sigma_{2}^{2}}{2 b_{21}} \\
& +\frac{a_{13}}{b_{31}}\left(-b_{3}+b_{31} \bar{S}\right) Y \leq-a_{11}(S-\bar{S})^{2}+K(\sigma),
\end{aligned}
$$

according to $b_{3} / b_{31} \geq b_{2} / b_{21}$. Integrating both sides of it from 0 to $t$ yields

$$
\begin{gathered}
V(t)-V(0) \leq-a_{11} \int_{0}^{t}(S(s)-S) \\
+\int_{0}^{t} \sigma_{1}(S(s)-\bar{S}) d B_{1}(s) \\
+\frac{a_{12}}{b_{21}} \sigma_{2}(X(s)-\bar{X}) d B_{2}(s) \\
+\frac{a_{13}}{b_{31}} \sigma_{3} Y(s) d B_{3}(s) .
\end{gathered}
$$

Let

$$
\begin{gathered}
M(t)=\int_{0}^{t} \sigma_{1}(S(s)-\bar{S}) d B_{1}(s) \\
+\frac{a_{12}}{b_{21}} \sigma_{2}(X(s)-\bar{X}) d B_{2}(s) \\
+\frac{a_{13}}{b_{31}} \sigma_{3} Y(s) d B_{3}(s),
\end{gathered}
$$

which is a real-valued continuous local martingale, and $M(0)=0$.

By Lemma 2, for arbitrary small $0<\varepsilon<1 / 2-1 / \theta$, there exists a constant $T=T(\omega)$ and a set $\Omega_{\varepsilon}$ such that $P\left(\Omega_{\varepsilon}\right) \geq 1-\varepsilon$ and, for $t \geq T, \omega \in \Omega_{\varepsilon}$,

$$
U(t) \leq t^{1 / \theta+\varepsilon} .
$$

Then,

$$
\begin{aligned}
& \int_{0}^{\infty} \frac{d\langle M, M\rangle_{t}}{(1+t)^{2}} \\
& \leq \int_{0}^{\infty} \frac{\sigma_{1}^{2}(S-\bar{S})^{2}+\left(a_{12}^{2} / b_{21}^{2}\right) \sigma_{2}^{2}(X-\bar{X})^{2}+\left(a_{13}^{2} / b_{31}^{2}\right) \sigma_{3}^{2} Y^{2}}{(1+t)^{2}} d t \\
& \leq 2 \int_{0}^{\infty} \frac{U^{2}}{(1+t)^{2}} d t+2 \int_{0}^{\infty} \frac{\sigma_{1}^{2} \bar{S}^{2}+\left(a_{12}^{2} / b_{21}^{2}\right) \sigma_{2}^{2} \bar{X}^{2}}{(1+t)^{2}} d t<\infty
\end{aligned}
$$


Let $\varepsilon \longrightarrow 0$; by the strong law of large numbers [16],

$$
\lim _{t \rightarrow \infty} \frac{M(t)}{t}=0 \quad \text { a.s. }
$$

It then follows from (32) that

$$
\limsup _{t \rightarrow \infty} \frac{1}{t} \int_{0}^{t}(S(s)-\bar{S})^{2} d s \leq \frac{K(\sigma)}{a_{11}} \quad \text { a.s. }
$$

By Itô's formula, we have

$$
\begin{aligned}
d \log Y= & \left(-b_{3}+b_{31} S-\frac{1}{2} \sigma_{3}^{2}\right) d t+\sigma_{3} d B_{3}(t) \\
= & {\left[-b_{3}-\frac{1}{2} \sigma_{3}^{2}+b_{31} \bar{S}+b_{31}(S-\bar{S})\right] d t } \\
& +\sigma_{3} d B_{3}(t)
\end{aligned}
$$

and then

$$
\begin{aligned}
\frac{\log Y(t)}{t}= & \frac{\log Y(0)}{t}-b_{3}-\frac{1}{2} \sigma_{3}^{2}+b_{31} \bar{S} \\
& +b_{31}\langle S(t)-\bar{S}\rangle+\frac{\sigma_{3} B_{3}(t)}{t} \\
\leq & \frac{\log Y(0)}{t}-b_{3}-\frac{1}{2} \sigma_{3}^{2}+b_{31} \bar{S} \\
& +b_{31}\left[\frac{1}{t} \int_{0}^{t}(S(s)-\bar{S})^{2} d s\right]^{1 / 2} \\
& +\frac{\sigma_{3} B_{3}(t)}{t}
\end{aligned}
$$

which together with $\lim _{t \rightarrow \infty} B_{3}(t) / t=0$ and (37) implies

$$
\begin{aligned}
\limsup _{t \rightarrow \infty} \frac{\log Y(t)}{t} \leq & -b_{3}-\frac{1}{2} \sigma_{3}^{2}+\frac{b_{31} b_{2}}{b_{21}} \\
& +b_{31}\left(\frac{K(\sigma)}{a_{11}}\right)^{1 / 2} \text { a.s. }
\end{aligned}
$$

From the condition $\left(b_{3}+(1 / 2) \sigma_{3}^{2}\right) / b_{31}>b_{2} / b_{21}+\left(K(\sigma) / a_{11}\right)^{1 / 2}$, it is easy to see that

$$
\begin{aligned}
\limsup _{t \rightarrow \infty} \frac{\log Y(t)}{t} \leq & -b_{3}-\frac{1}{2} \sigma_{3}^{2}+\frac{b_{31} b_{2}}{b_{21}} \\
& +b_{31}\left(\frac{K(\sigma)}{a_{11}}\right)^{1 / 2}<0 \quad \text { a.s.; }
\end{aligned}
$$

namely, $Y(t)$ tends to zero exponentially almost surely.

We derive from (2) that

$$
\begin{aligned}
\frac{\log S(t)}{t}= & \frac{\log S(0)}{t}+b_{1}-\frac{1}{2} \sigma_{1}^{2}-a_{11}\langle S(t)\rangle \\
& -a_{12}\langle X(t)\rangle-a_{13}\langle Y(t)\rangle+\frac{\sigma_{1} B_{1}(t)}{t}
\end{aligned}
$$

and

$\frac{\log X(t)}{t}=\frac{\log X(0)}{t}-b_{2}-\frac{1}{2} \sigma_{2}^{2}+b_{21}\langle S(t)\rangle+\frac{\sigma_{2} B_{2}(t)}{t}$.

From Lemma 2, we know that

$$
\limsup _{t \rightarrow \infty} \frac{\log U(t)}{t} \leq 0 \quad \text { a.s. }
$$

which implies

$$
\begin{gathered}
\underset{t \rightarrow \infty}{\limsup \frac{\log X(t)}{t} \leq \limsup _{t \rightarrow \infty} \frac{\log U(t)}{t} \leq 0 \quad \text { a.s. }} \\
\limsup _{t \rightarrow \infty} \frac{\log S(t)}{t} \leq \limsup _{t \rightarrow \infty} \frac{\log U(t)}{t} \leq 0 \quad \text { a.s. }
\end{gathered}
$$

Substituting (45) into (43) yields that

$$
\limsup _{t \rightarrow \infty}\langle S(t)\rangle \leq \frac{b_{2}+(1 / 2) \sigma_{2}^{2}}{b_{21}} \quad \text { a.s. }
$$

It then follows from (42), (46), and (47), using $\lim _{t \rightarrow \infty}\langle Y(t)\rangle=0$ and the condition $\left(b_{1}-(1 / 2) \sigma_{1}^{2}\right) / a_{11}>$ $\left(b_{2}+(1 / 2) \sigma_{2}^{2}\right) / b_{21}$ as well, that

$$
\begin{aligned}
\liminf _{t \rightarrow \infty}\langle X(t)\rangle & \geq \frac{1}{a_{12}}\left[b_{1}-\frac{1}{2} \sigma_{1}^{2}-a_{11} \frac{b_{2}+(1 / 2) \sigma_{2}^{2}}{b_{21}}\right] \\
& >0 \quad \text { a.s. }
\end{aligned}
$$

(ii) The proof is similar to the former part of the proof.

Theorem 4. Let $(S(t), X(t), Y(t))$ be the solution of system (2) with initial value $(S(0), X(0), Y(0)) \in R_{+}^{3}$. The principle of competitive exclusion does not hold for system (2) if $b_{1}>$ $(1 / 2) \sigma_{1}^{2},\left(b_{1}-(1 / 2) \sigma_{1}^{2}\right) / a_{11}<\left(b_{2}+(1 / 2) \sigma_{2}^{2}\right) / b_{21}$, and $\left(b_{1}-\right.$ $\left.(1 / 2) \sigma_{1}^{2}\right) / a_{11}<\left(b_{3}+(1 / 2) \sigma_{3}^{2}\right) / b_{31}$. In this case

$$
\begin{aligned}
\lim _{t \rightarrow \infty}\langle S(t)\rangle & =\frac{b_{1}-(1 / 2) \sigma_{1}^{2}}{a_{11}} \quad \text { a.s. } \\
\limsup _{t \rightarrow \infty} \frac{\log X(t)}{t} & \leq-b_{2}-\frac{1}{2} \sigma_{2}^{2}+b_{21} \frac{b_{1}-(1 / 2) \sigma_{1}^{2}}{a_{11}}<0
\end{aligned}
$$

$$
\limsup _{t \rightarrow \infty} \frac{\log Y(t)}{t} \leq-b_{3}-\frac{1}{2} \sigma_{3}^{2}+b_{31} \frac{b_{1}-(1 / 2) \sigma_{1}^{2}}{a_{11}}<0
$$

In addition,

$$
\begin{aligned}
& \lim _{t \rightarrow \infty} S(t)=0, \\
& \lim _{t \rightarrow \infty} X(t)=0 \text { and } \\
& \lim _{t \rightarrow \infty} Y(t)=0
\end{aligned}
$$

a.s. 
Proof. Since the solution of system (2) is positive, by a classical comparison theorem of stochastic differential equations, it is clear that $S(t) \leq \phi(t)$ a.s., where $\phi(t)$ is the solution of the stochastic logistic equation:

$$
d \phi(t)=\phi(t)\left(b_{1}-a_{11} \phi(t)\right) d t+\sigma_{1} \phi(t) d B_{1}(t) .
$$

From the result in [17], we know

$$
\begin{aligned}
& \lim _{t \rightarrow \infty}\langle\phi(t)\rangle=\frac{b_{1}-\sigma_{1}^{2} / 2}{a_{11}}, \\
& \lim _{t \rightarrow \infty} \frac{\log \phi(t)}{t}=0
\end{aligned}
$$

a.s.

provided $b_{1}>\sigma_{1}^{2} / 2$.

By Itô's formula, we have

$$
\begin{aligned}
d \log X & =\left(-b_{2}+b_{21} S-\frac{\sigma_{2}^{2}}{2}\right) d t+\sigma_{2} d B_{2}(t) \\
& \leq\left(-b_{2}+b_{21} \phi-\frac{\sigma_{2}^{2}}{2}\right) d t+\sigma_{2} d B_{2}(t)
\end{aligned}
$$

and then

$$
\begin{aligned}
\frac{\log X(t)}{t} \leq & \frac{\log X(0)}{t}-b_{2}-\frac{\sigma_{2}^{2}}{2}+b_{21}\langle\phi(t)\rangle \\
& +\sigma_{2} \frac{B_{2}(t)}{t} .
\end{aligned}
$$

From (52) and the condition $\left(b_{1}-(1 / 2) \sigma_{1}^{2}\right) / a_{11}<\left(b_{2}+\right.$ $\left.(1 / 2) \sigma_{2}^{2}\right) / b_{21}$, it is easy to see that

$$
\limsup _{t \rightarrow \infty} \frac{\log X(t)}{t} \leq-b_{2}-\frac{\sigma_{2}^{2}}{2}+b_{21} \frac{b_{1}-(1 / 2) \sigma_{1}^{2}}{a_{11}}<0
$$

a.s.;

namely, $X(t)$ tends to zero exponentially almost surely.

By Itô's formula, we have

$$
\begin{aligned}
d \log Y & =\left(-b_{3}+b_{31} S-\frac{\sigma_{3}^{2}}{2}\right) d t+\sigma_{3} d B_{3}(t) \\
& \leq\left(-b_{3}+b_{31} \phi-\frac{\sigma_{3}^{2}}{2}\right) d t+\sigma_{3} d B_{3}(t),
\end{aligned}
$$

so

$$
\begin{aligned}
\frac{\log Y(t)}{t} \leq & \frac{\log Y(0)}{t}-b_{3}-\frac{\sigma_{3}^{2}}{2}+b_{31}\langle\phi(t)\rangle \\
& +\sigma_{3} \frac{B_{3}(t)}{t} .
\end{aligned}
$$

Together with (52) and the condition $\left(b_{1}-(1 / 2) \sigma_{1}^{2}\right) / a_{11}<$ $\left(b_{3}+(1 / 2) \sigma_{3}^{2}\right) / b_{31}$, we have

$$
\limsup _{t \rightarrow \infty} \frac{\log Y(t)}{t} \leq-b_{3}-\frac{\sigma_{3}^{2}}{2}+b_{31} \frac{b_{1}-(1 / 2) \sigma_{1}^{2}}{a_{11}}<0
$$

namely, $Y(t)$ tends to zero exponentially almost surely. We derive from (42) that

$$
\begin{aligned}
a_{11}\langle S(t)\rangle= & b_{1}-\frac{\sigma_{1}^{2}}{2}-\frac{\log S(t)-\log S(0)}{t} \\
& -a_{12}\langle X(t)\rangle-a_{13}\langle Y(t)\rangle+\sigma_{1} \frac{B_{1}(t)}{t} \\
\geq & b_{1}-\frac{\sigma_{1}^{2}}{2}-\frac{\log \phi(t)-\log S(0)}{t} \\
& -a_{12}\langle X(t)\rangle-a_{13}\langle Y(t)\rangle+\sigma_{1} \frac{B_{1}(t)}{t} .
\end{aligned}
$$

This together with (52), (55), and (58) implies that

$$
\liminf _{t \rightarrow \infty}\langle S(t)\rangle \geq \frac{b_{1}-\sigma_{1}^{2} / 2}{a_{11}} \text { a.s. }
$$

From

$$
\begin{aligned}
\frac{b_{1}-\sigma_{1}^{2} / 2}{a_{11}} & \leq \liminf _{t \longrightarrow \infty}\langle S(t)\rangle \leq \limsup _{t \rightarrow \infty}\langle S(t)\rangle \\
& \leq \limsup _{t \rightarrow \infty}\langle\phi(t)\rangle=\frac{b_{1}-\sigma_{1}^{2} / 2}{a_{11}} \quad \text { a.s. }
\end{aligned}
$$

we obtain

$$
\lim _{t \rightarrow \infty}\langle S(t)\rangle=\frac{b_{1}-\sigma_{1}^{2} / 2}{a_{11}} \text { a.s. }
$$

If $b_{1}<\frac{1}{2} \sigma_{1}^{2}$, it is easy to see that

$$
\begin{aligned}
& \lim _{t \rightarrow \infty} S(t)=0, \\
& \lim _{t \rightarrow \infty} X(t)=0 \text { and } \\
& \lim _{t \rightarrow \infty} Y(t)=0
\end{aligned}
$$

from (52), (55), and (58).

Remark 5. By Lemma 2.5 in [12], they study the persistence and extinction of each species, to reveal the effects of stochastic noises on the persistence and extinction of each species. However, we give the sufficient conditions to guarantee that the principle of competitive exclusion holds for the model by constructing suitable stochastic Lyapunov functions, which is the biggest difference between this paper and [12]. 


\section{Numerical Simulations}

We present some examples to confirm and visualize the observed results by using Milstein's Higher Order Method [18].

Example 1. Choose parameters $b_{1}=1, b_{2}=1.2, b_{3}=$ $1.5, a_{11}=0.3, a_{12}=0.2, a_{13}=0.3, b_{21}=0.4, b_{31}=$ 0.3 . These values imply that $b_{2} / b_{21}<b_{1} / a_{11}<b_{3} / b_{31}$. For deterministic system (1), the predator species $X(t)$ survives, and the predator species $Y(t)$ goes to extinction.

Next, we observe the role of competition in the stochastic system (2) under environmental disturbance.

(i) We take $\sigma_{1}=0.1, \sigma_{2}=0.1, \sigma_{3}=0.1$. In this case,

$$
\begin{aligned}
1 & =b_{1}>\frac{1}{2} \sigma_{1}^{2}=0.005, \\
5 & =\frac{b_{3}}{b_{31}} \geq \frac{b_{2}}{b_{21}}=3, \\
5.017 & =\frac{b_{3}+(1 / 2) \sigma_{3}^{2}}{b_{31}}>\frac{b_{2}}{b_{21}}+\left(\frac{K(\sigma)}{a_{11}}\right)^{1 / 2}=3.23
\end{aligned}
$$

and

$$
3.317=\frac{b_{1}-(1 / 2) \sigma_{1}^{2}}{a_{11}}>\frac{b_{2}+(1 / 2) \sigma_{2}^{2}}{b_{21}}=3.0125 .
$$

We conclude from Theorem 3, for the initial value $S(0)=$ $1, X(0)=1, Y(0)=1$, that the solution of system (2) obeys

$$
\begin{aligned}
& \liminf _{t \rightarrow \infty}\langle X(t)\rangle \geq \frac{1}{a_{12}}\left[b_{1}-\frac{1}{2} \sigma_{1}^{2}-a_{11} \frac{b_{2}+(1 / 2) \sigma_{2}^{2}}{b_{21}}\right] \\
& \quad=0.45625>0 \text { a.s. } \\
& \limsup _{t \rightarrow \infty} \frac{\log Y(t)}{t} \\
& \quad \leq-b_{3}-\frac{1}{2} \sigma_{3}^{2}+\frac{b_{31} b_{2}}{b_{21}}+b_{31}\left(\frac{K(\sigma)}{a_{11}}\right)^{1 / 2}=-0.536 \\
& \quad<0 \quad \text { a.s. }
\end{aligned}
$$

The numerical simulations in Figure 1 support these results clearly, illustrating survival of the predator species $X(t)$ and extinction of the predator species $Y(t)$.

Furthermore, we choose the same parameters as in Figure 1 but change the intensities of the white noise $\left(\sigma_{1}^{2}=\right.$ $\sigma_{2}^{2}=\sigma_{3}^{2}=0.1^{2}$ and $\sigma_{1}^{2}=\sigma_{2}^{2}=\sigma_{3}^{2}=0.06^{2}$ ), which also satisfy the conditions in Theorem 3 . As expected, Figures 2 and 3 show the solution (the red lines) is fluctuating around a small zone. By comparing Figures 2 and 3, we can see with a decrease of the white noise, the zone which the solution is fluctuating in is getting small. From their density functions (on the right of this figure), we consider that $(S(t), X(t))$ has a stationary distribution. (ii) We choose $\sigma_{1}=0.1, \sigma_{2}=0.6, \sigma_{3}=0.3$. The conditions in Theorem 4

$$
\begin{aligned}
1 & =b_{1}>\frac{1}{2} \sigma_{1}^{2}=0.005 \\
3.317 & =\frac{b_{1}-(1 / 2) \sigma_{1}^{2}}{a_{11}}<\frac{b_{2}+(1 / 2) \sigma_{2}^{2}}{b_{21}}=3.45
\end{aligned}
$$

and

$$
3.317=\frac{b_{1}-(1 / 2) \sigma_{1}^{2}}{a_{11}}<\frac{b_{3}+(1 / 2) \sigma_{3}^{2}}{b_{31}}=5.15
$$

are satisfied. In this case,

$$
\begin{aligned}
\lim _{t \rightarrow \infty}\langle S(t)\rangle & =\frac{b_{1}-(1 / 2) \sigma_{1}^{2}}{a_{11}}=3.317 \text { a.s. } \\
\limsup _{t \rightarrow \infty} \frac{\log X(t)}{t} & \leq-b_{2}-\frac{1}{2} \sigma_{2}^{2}+b_{21} \frac{b_{1}-(1 / 2) \sigma_{1}^{2}}{a_{11}} \\
& =-0.0533<0 \quad \text { a.s. } \\
\limsup _{t \rightarrow \infty} \frac{\log Y(t)}{t} & \leq-b_{3}-\frac{1}{2} \sigma_{3}^{2}+b_{31} \frac{b_{1}-(1 / 2) \sigma_{1}^{2}}{a_{11}} \\
& =-0.5499<0 \quad \text { a.s. }
\end{aligned}
$$

The numerical simulations, in Figure 4, support these results clearly, illustrating extinction of the competing predator species $X(t)$ and $Y(t)$.

(iii) We choose $\sigma_{1}=1.5, \sigma_{2}=0.8, \sigma_{3}=1.0$. In this case, $1=b_{1}<(1 / 2) \sigma_{1}^{2}=1.125$. Then

$$
\begin{aligned}
& \lim _{t \rightarrow \infty} S(t)=0, \\
& \lim _{t \rightarrow \infty} X(t)=0 \text { and } \\
& \lim _{t \rightarrow \infty} Y(t)=0 .
\end{aligned}
$$

The numerical simulations in Figure 5 support these results clearly, illustrating extinction of the prey species $S(t)$ and the competing predator species $X(t), Y(t)$.

Example 2. Choose parameters $b_{1}=1.5, b_{2}=0.9, b_{3}=$ $1.2, a_{11}=0.3, a_{12}=0.2, a_{13}=0.3, b_{21}=0.3, b_{31}=$ 0.4 . These values imply that $b_{2} / b_{21}=b_{3} / b_{31}<b_{1} / a_{11}$. For deterministic system (1), two competing predators coexist at a positive equilibrium.

However, we choose $\sigma_{1}=0.1, \sigma_{2}=0.1, \sigma_{3}=0.6$. The conditions in Theorem 3

$$
\begin{gathered}
1.5=b_{1}>\frac{1}{2} \sigma_{1}^{2}=0.005, \\
3=\frac{b_{3}}{b_{31}} \geq \frac{b_{2}}{b_{21}}=3,
\end{gathered}
$$



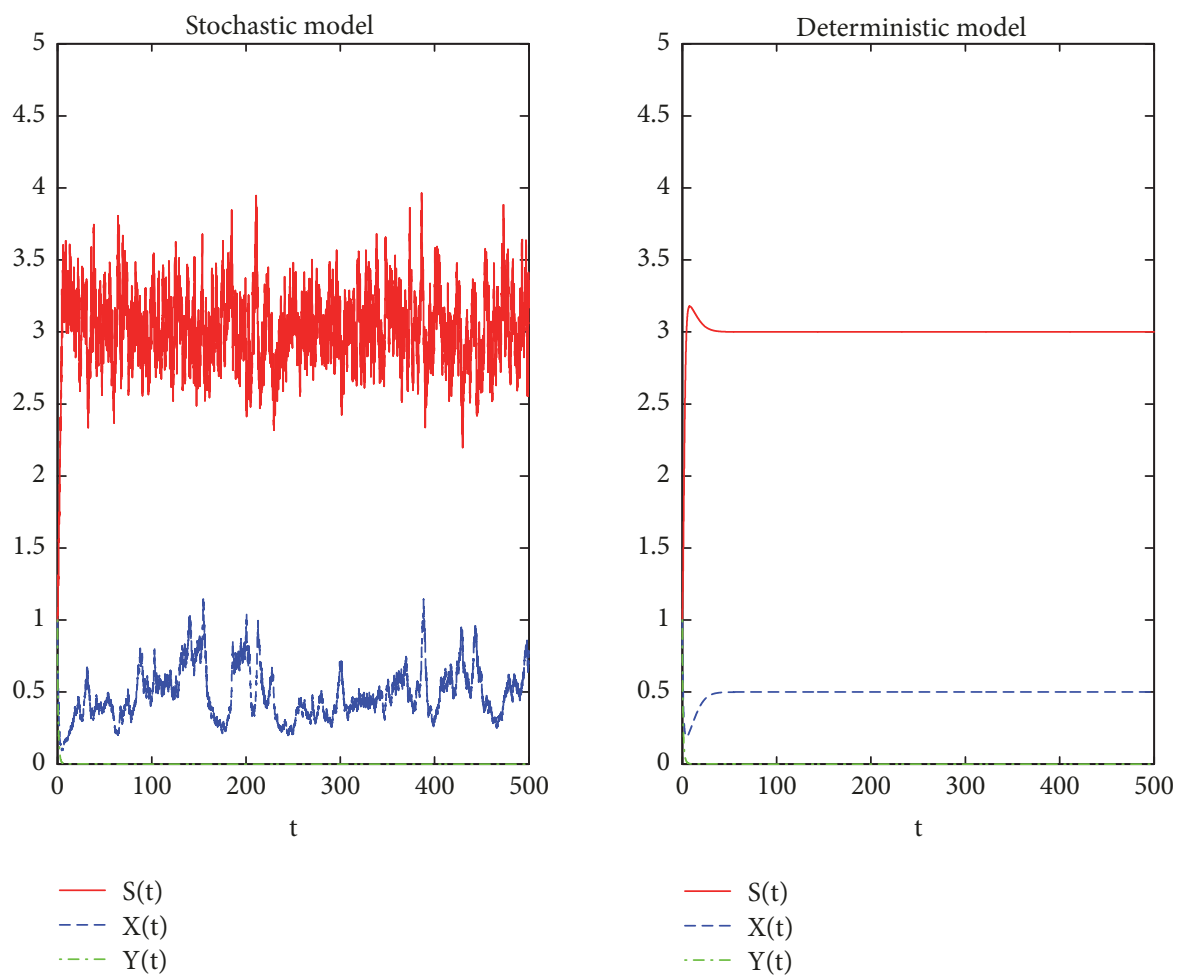

FIGURE 1: Numerical simulation of the solution of system (2) and its corresponding deterministic system (1) with $\sigma_{1}=0.1, \sigma_{2}=0.1, \sigma_{3}=0.1$, with initial value $S(0)=1, X(0)=1, Y(0)=1$.
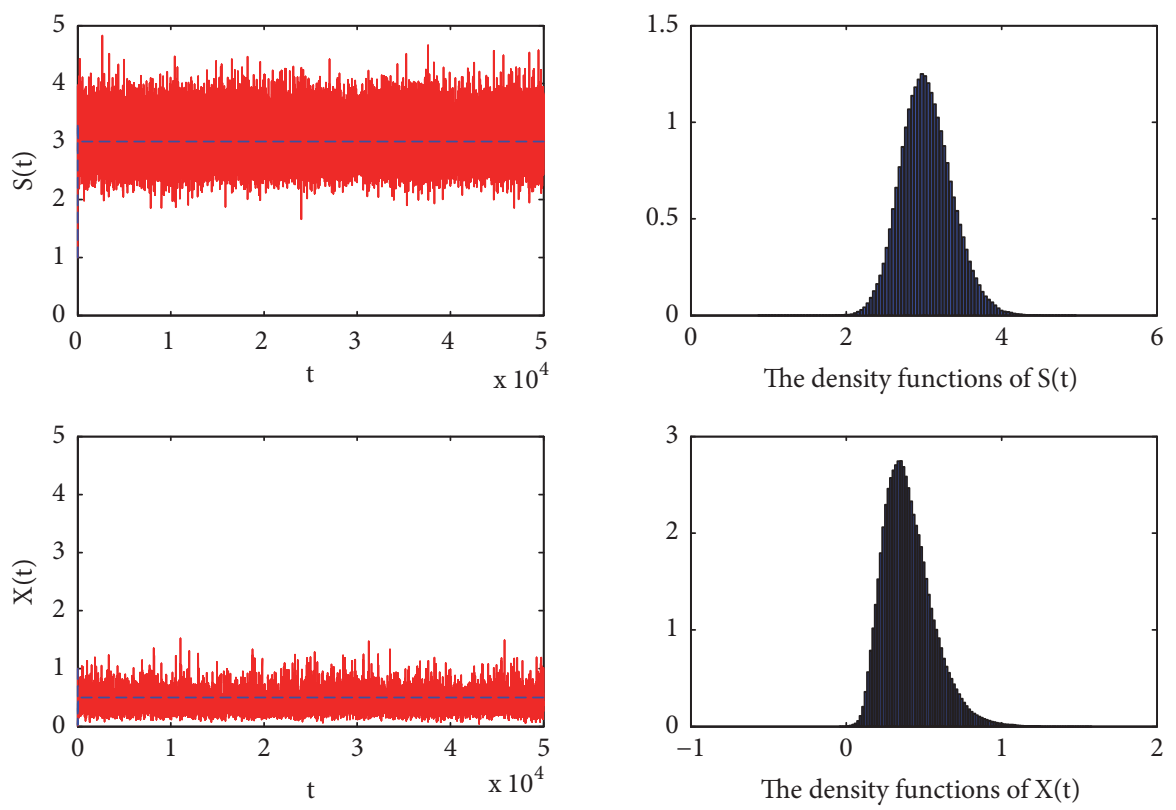

Figure 2: $(S(t), X(t))$ has a stationary distribution. In the left, the red lines represent the solution of system (2), and the blue lines represent the solution of the corresponding undisturbed system. The pictures on the right are the density functions of system (2).

$$
\begin{aligned}
3.45 & =\frac{b_{3}+(1 / 2) \sigma_{3}^{2}}{b_{31}}>\max \left\{\frac{b_{2}}{b_{21}}+\left(\frac{K(\sigma)}{a_{11}}\right)^{1 / 2}\right\} & \text { and } \\
& =3.28 & (71) \quad 4.983=\frac{b_{1}-(1 / 2) \sigma_{1}^{2}}{a_{11}}>\frac{b_{2}+(1 / 2) \sigma_{2}^{2}}{b_{21}}=3.017
\end{aligned}
$$



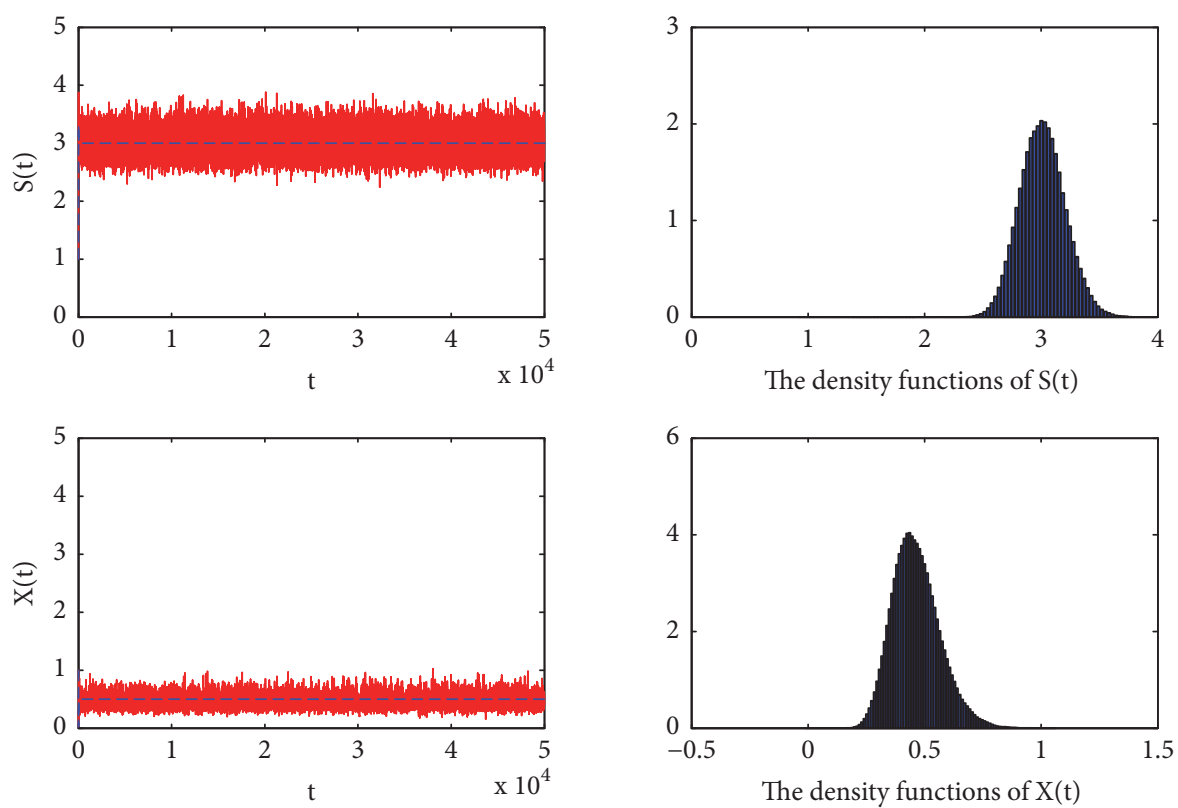

Figure 3: There also exists a stationary distribution of $(S(t), X(t))$, and the fluctuation is reduced with the decreasing of the white noise. The lines have the same meaning as Figure 2 .
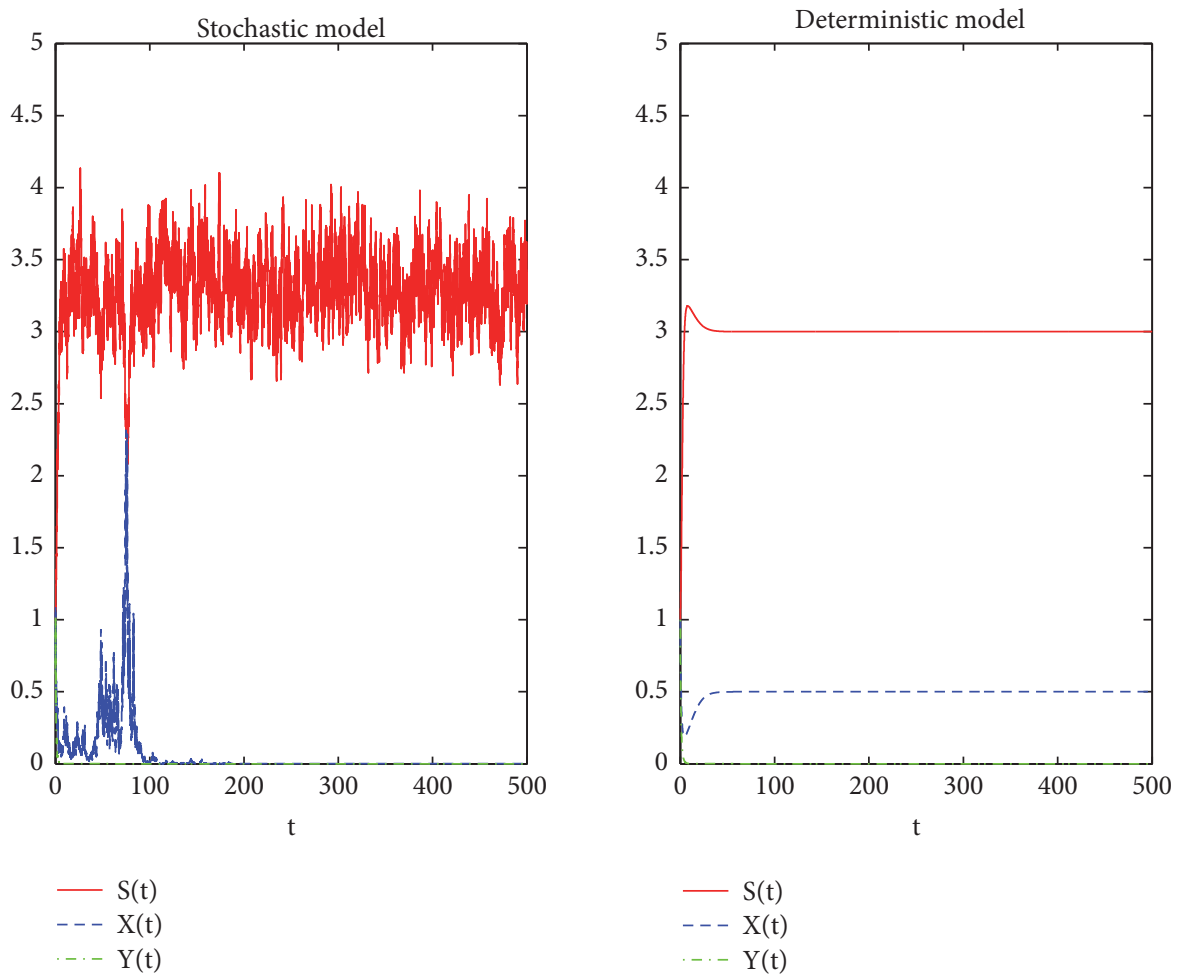

FIGURE 4: Numerical simulation of the solution of system (2) and its corresponding deterministic system (1) with $\sigma_{1}=0.1, \sigma_{2}=0.6, \sigma_{3}=0.3$, with initial value $S(0)=1, X(0)=1, Y(0)=1$.

are satisfied. For stochastic system (2), the numerical simulations in Figure 6, support these results clearly, illustrating survival of the predator species $X(t)$ and extinction of the predator species $Y(t)$.

\section{Conclusion}

In this paper, we have proposed and analyzed the principle of competitive exclusion about a Lotka-Volterra model with two predators competing for one prey by stochastic perturbation. 

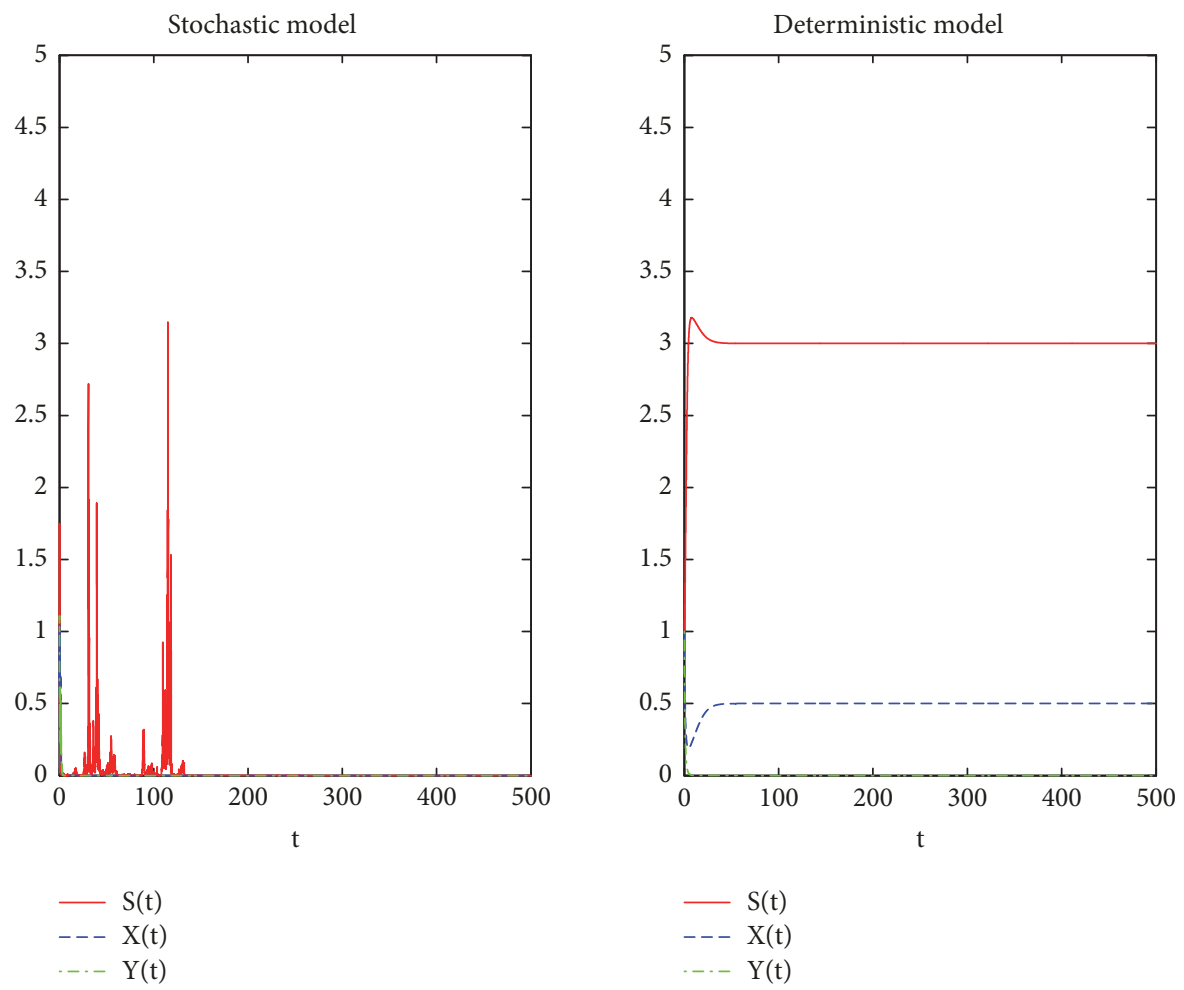

FIGURE 5: Numerical simulation of the solution of system (2) and its corresponding deterministic system (1) with $\sigma_{1}=1.5, \sigma_{2}=0.8, \sigma_{3}=1.0$, with initial value $S(0)=1, X(0)=1, Y(0)=1$.
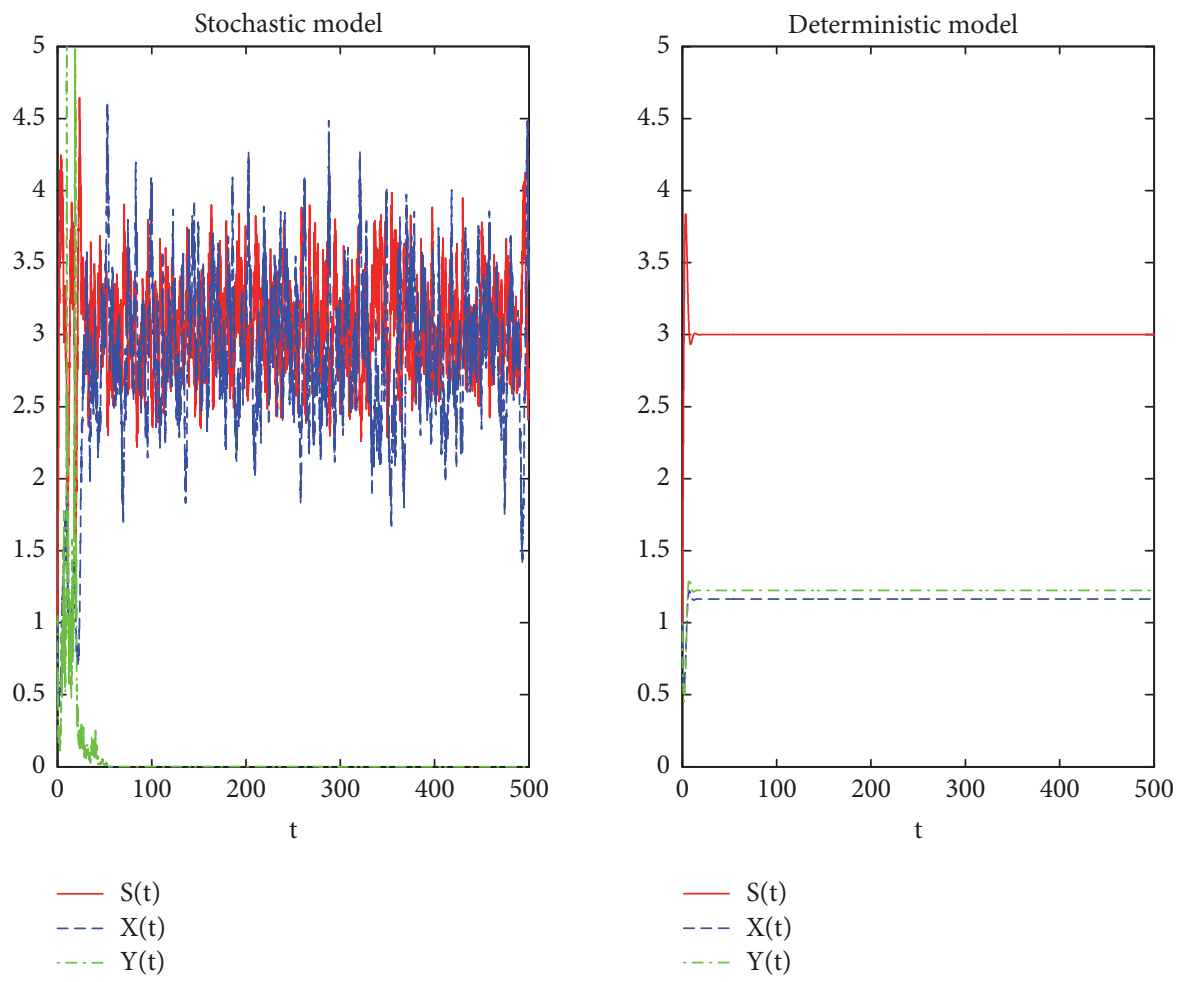

FiguRE 6: Numerical simulation of the solution of system (2) and its corresponding deterministic system (1) with $\sigma_{1}=0.1, \sigma_{2}=0.1, \sigma_{3}=0.6$, with initial value $S(0)=1, X(0)=1, Y(0)=1$. 
Based on this model, we mainly have investigated that system (2) allowed the competitive exclusion of two competing predators for some values of parameters when the predator functional response to the prey density is linear. Theorem 3 shows that when $\sigma_{1}^{2}<2 b_{1}$, the principle of competitive exclusion holds for system (2) under certain conditions. In this case, the predator species $Y(t)$ (or $X(t)$ ) goes to extinction, and the predator species $X(t)$ (or $Y(t)$ ) survives. Theorem 4 shows that if $\sigma_{1}^{2}<2 b_{1}$, the principle of competitive exclusion does not hold for system (2) under certain conditions. In this case, both competing predators go to extinction. If $\sigma_{1}^{2}>$ $2 b_{1}$, it is easy to see that $\lim _{t \rightarrow \infty} S(t)=0, \lim _{t \rightarrow \infty} X(t)=$ 0 and $\lim _{t \rightarrow \infty} Y(t)=0$. Furthermore, from the numerical simulation, if conditions about the principle of competitive exclusion hold, we can see that there exists a stationary distribution of $(S(t), X(t))$ for this system when the white noise is small.

In this paper, we only considered the white noise. In fact, there are some random perturbations, for example, the telephone noise. Recently, stochastic models with the telephone noise have been studied by many authors; see [1922]. In the future study, we will consider a stochastic LotkaVolterra model with two predators competing for one prey perturbed by the telephone noise.

\section{Data Availability}

The data used to support the findings of this study are available from the corresponding author upon request.

\section{Conflicts of Interest}

The authors declare that they have no conflicts of interest.

\section{Acknowledgments}

The work was supported by Program for Natural Science Foundation of China (nos. 11701209 and 11601038), the natural science foundation of Jilin Province (no. 20170101146JC), Science and Technology Research Project of Jilin Provincial Department of Education (no. JJKH20170487KJ), Youth Science Foundation of Jilin Province (no. 20160520110JH), Education Department of Jilin Province (no. JJKH20180462KJ), and Youth Teacher Development Program of Changchun University (no. 2018JBC08L13).

\section{References}

[1] A. L. Koch, "Competitive coexistence of two predators utilizing the same prey under constant environmental conditions," Journal of Theoretical Biology, vol. 44, no. 2, pp. 387-395, 1974.

[2] S. B. Hsu, S. P. Hubbell, and P. Waltman, "Competing predators," SIAM Journal on Applied Mathematics, vol. 35, no. 4, pp. 617625, 1978.

[3] S. Hsu, S. Hubbell, and P. Waltman, "A contribution to the theory of competing predators," Ecological Monographs, vol. 48, pp. 337-349, 1978.

[4] A. Korobeinikov and G. Wake, "Global properties of the threedimensional predator-prey Lotka-Volterra systems," Journal of
Applied Mathematics and Decision Sciences, vol. 3, pp. 155-162, 1999.

[5] B. Li and H. L. Smith, "Global dynamics of microbial competition for two resources with internal storage," Journal of Mathematical Biology, vol. 55, no. 4, pp. 481-515, 2007.

[6] J. Llibre and D. Xiao, "Global dynamics of a Lotka-Volterra model with two predators competing for one prey," SIAM Journal on Applied Mathematics, vol. 74, no. 2, pp. 434-453, 2014.

[7] W. Liu, D. Xiao, and Y. Yi, "Relaxation oscillations in a class of predator-prey systems," Journal of Differential Equations, vol. 188, no. 1, pp. 306-331, 2003.

[8] G. S. K. Wolkowicz and Z. Lu, "Global dynamics of a mathematical model of competition in the chemostat: general response functions and differential death rates," SIAM Journal on Applied Mathematics, vol. 52, no. 1, pp. 222-233, 1992.

[9] X. Li, D. Jiang, and X. Mao, "Population dynamical behavior of Lotka-Volterra system under regime switching," Journal of Computational and Applied Mathematics, vol. 232, no. 2, pp. 427-448, 2009.

[10] Y. Zhao, D. Jiang, and D. O'Regan, "The extinction and persistence of the stochastic SIS epidemic model with vaccination," Physica A: Statistical Mechanics and its Applications, vol. 392, no. 20, pp. 4916-4927, 2013.

[11] Y. Zhao and D. Jiang, "The threshold of a stochastic SIS epidemic model with vaccination," Applied Mathematics and Computation, vol. 243, pp. 718-727, 2014.

[12] M. Liu and P. S. Mandal, "Dynamical behavior of a one-prey two-predator model with random perturbations," Communications in Nonlinear Science and Numerical Simulation, vol. 28, no. 1-3, pp. 123-137, 2015.

[13] Q. Zhang, D. Jiang, Z. Liu, and D. O’Regan, “The long time behavior of a predator-prey model with disease in the prey by stochastic perturbation," Applied Mathematics and Computation, vol. 245, pp. 305-320, 2014.

[14] Q. Zhang and D. Jiang, "Competitive exclusion in a stochastic chemostat model with Holling type II functional response," Journal of Mathematical Chemistry, vol. 54, no. 3, pp. 777-791, 2016.

[15] Q. Zhang and D. Jiang, "The coexistence of a stochastic LotkaVolterra model with two predators competing for one prey," Applied Mathematics and Computation, vol. 269, Article ID 21456, pp. 288-300, 2015.

[16] X. Mao, Stochastic Differential Equations and Applications, Horwood, Chichester, UK, 2nd edition, 1997.

[17] C. Ji, D. Jiang, and N. Shi, "Analysis of a predator-prey model with modified Leslie-Gower and Holling-type II schemes with stochastic perturbation," Journal of Mathematical Analysis and Applications, vol. 359, no. 2, pp. 482-498, 2009.

[18] D. J. Higham, "An algorithmic introduction to numerical simulation of stochastic differential equations," SIAM Review, vol. 43, no. 3, pp. 525-546, 2001.

[19] D. Li, S. Liu, and J. Cui, "Threshold dynamics and ergodicity of an SIRS epidemic model with Markovian switching," Journal of Differential Equations, vol. 263, no. 12, pp. 8873-8915, 2017.

[20] M. Liu, X. He, and J. Yu, "Dynamics of a stochastic regimeswitching predator-prey model with harvesting and distributed delays," Nonlinear Analysis: Hybrid Systems, vol. 28, pp. 87-104, 2018.

[21] M. Liu and Y. Zhu, "Stability of a budworm growth model with random perturbations," Applied Mathematics Letters, vol. 79, pp. 13-19, 2018. 
[22] L. Wang and D. Jiang, "Ergodic property of the chemostat: a stochastic model under regime switching and with general response function," Nonlinear Analysis: Hybrid Systems, vol. 27, pp. 341-352, 2018. 


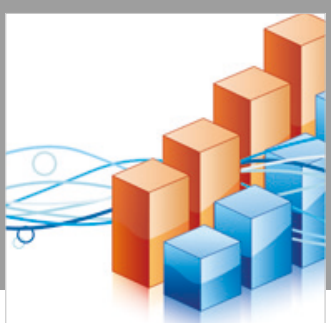

Advances in

Operations Research

\section{-n-m}
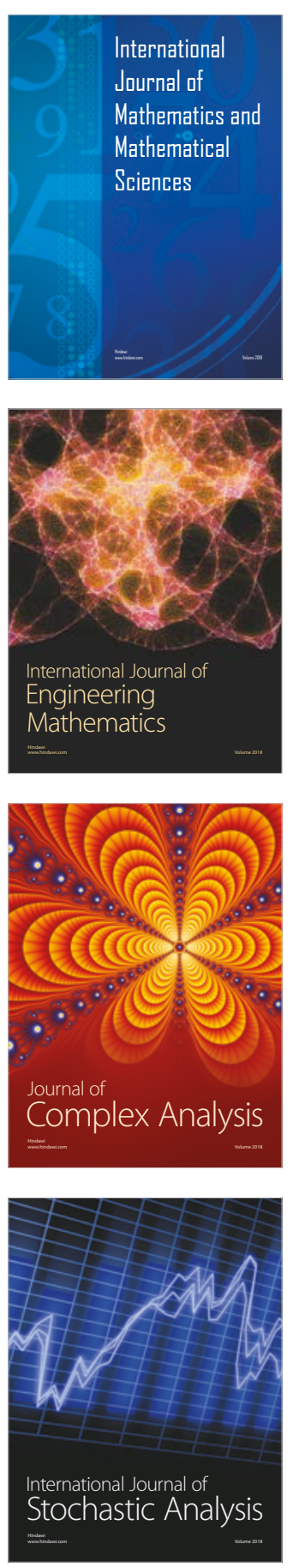
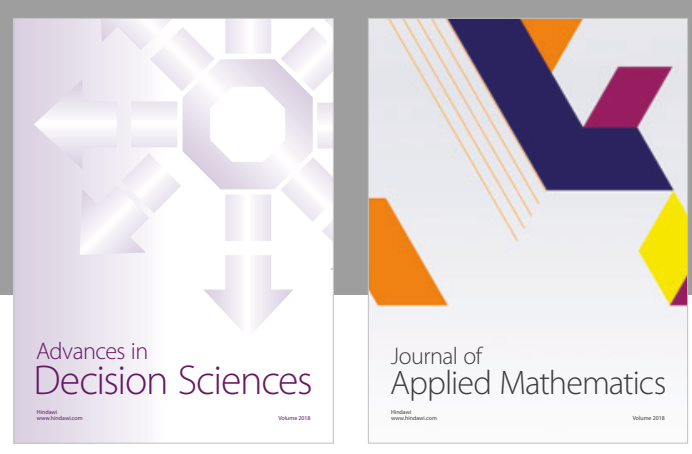

Journal of

Applied Mathematics
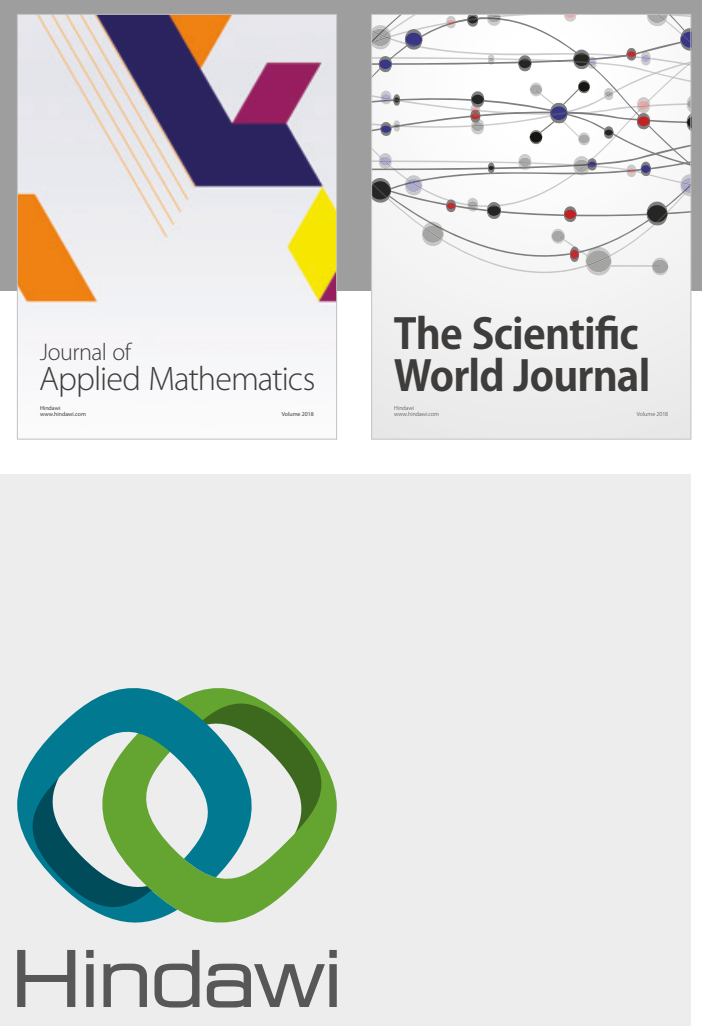

Submit your manuscripts at

www.hindawi.com

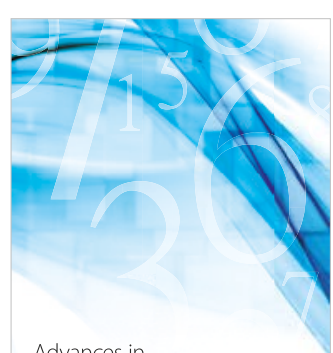

Advances in
Numerical Analysis
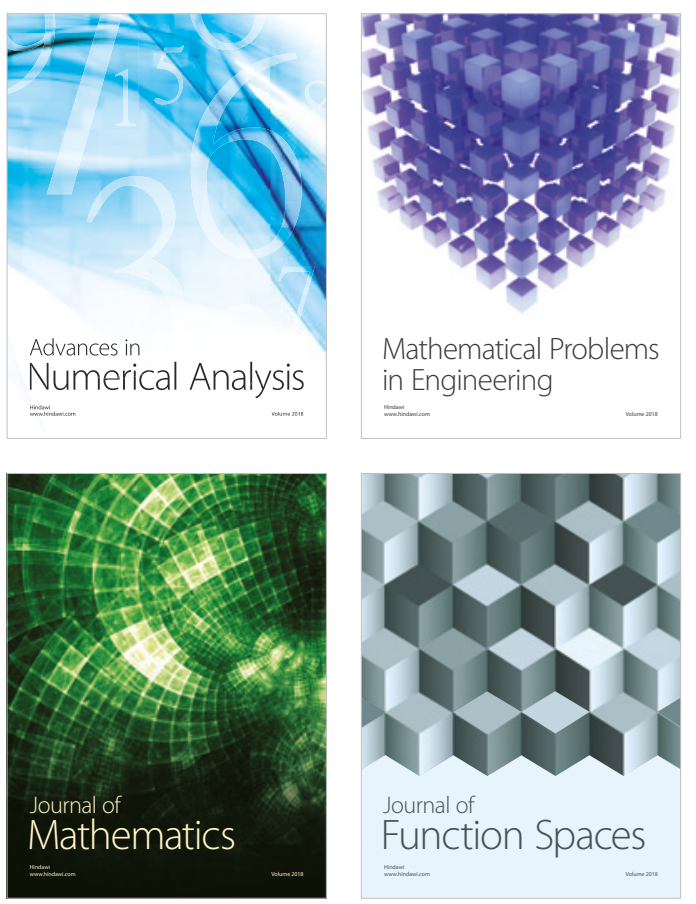

Mathematical Problems in Engineering

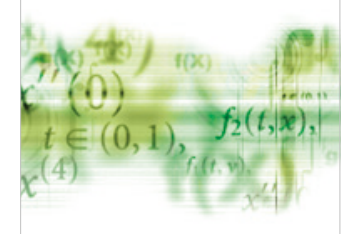

International Journal of

Differential Equations

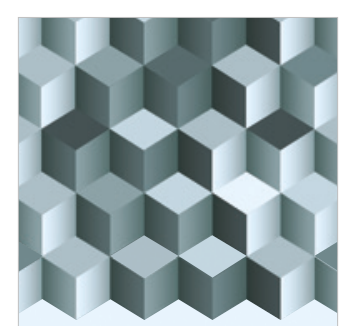

Journal of

Function Spaces

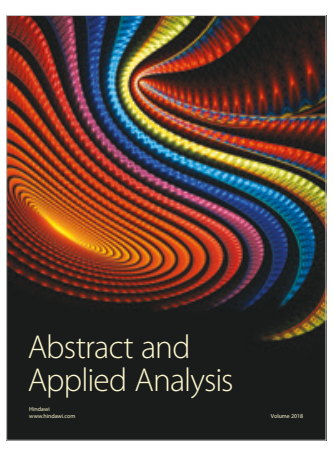

The Scientific

World Journal

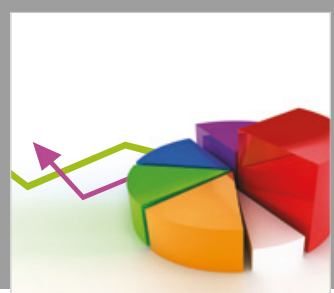

Journal of

Probability and Statistics
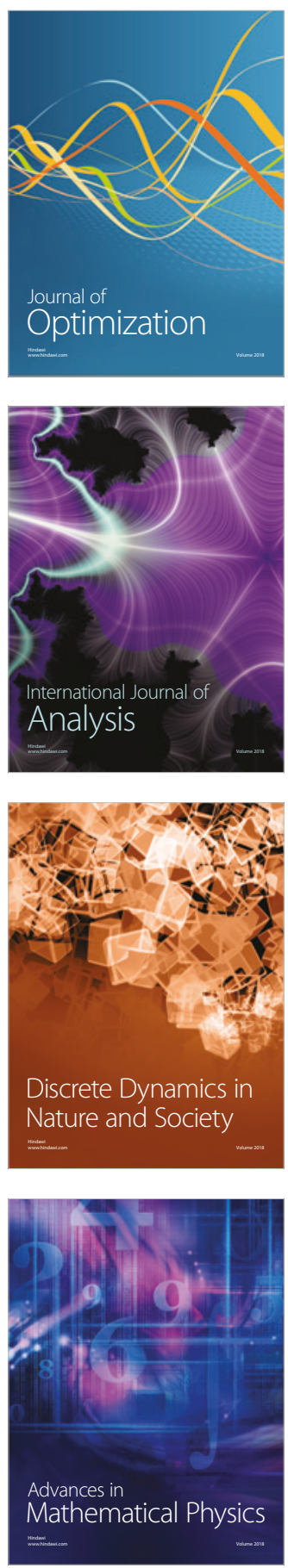